Supporting information for

\title{
Mass Spectrometry Imaging of Mass Tag Immunoassay Enables Quantitative Profiling of Biomarkers from Dozens of Exosomes
}

Yuning Wang, ${ }^{\mathrm{a}}$ Kun Zhang, ${ }^{\mathrm{b}}$ Xuedong Huang, ${ }^{\mathrm{a}}$ Liang Qiao* a and Baohong Liu* a

a. Department of Chemistry, Shanghai Stomatological Hospital, State Key Laboratory of Molecular Engineering of Polymers, Fudan University, Shanghai 200438, China.

*E-mail: bhliu@fudan.edu.cn; liang_qiao@,fudan.edu.cn.

b. Department of Neurosurgery, Shanghai Children's Hospital, Shanghai Jiao Tong University, Shanghai 200062, China. 
Table of Contents

Supplementary Experimental Section

Chemicals and materials...............................................................................

Cell culture and exosome isolation.................................................................S3

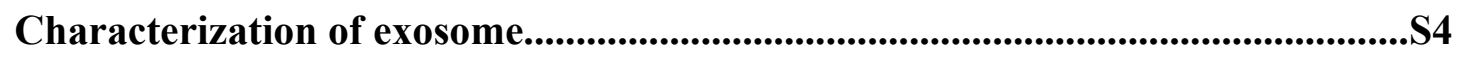

Screening exosome protein biomarkers by ELISA..........................................S4

\section{Supplementary Figure}

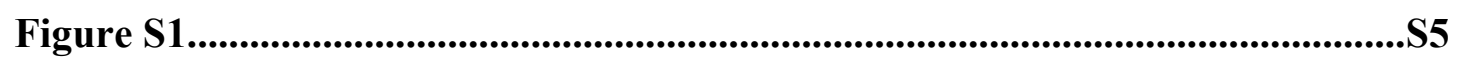

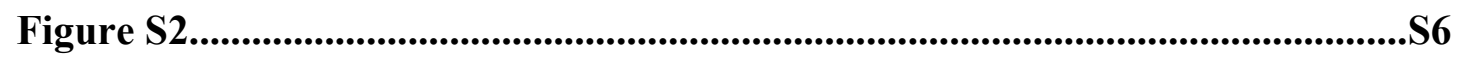

Figure $\mathbf{S 3}$..........................................................................................................................S7

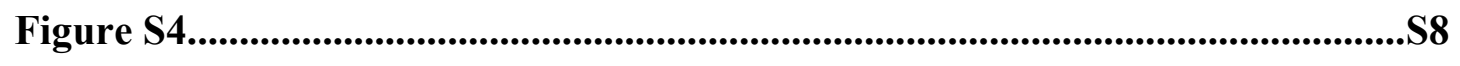

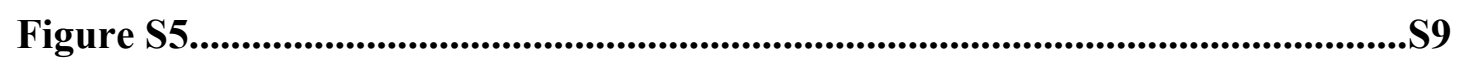

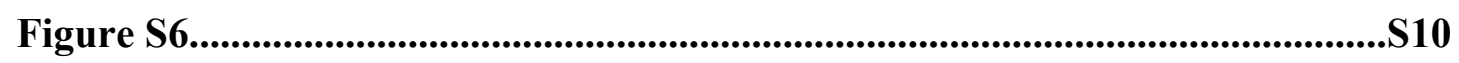

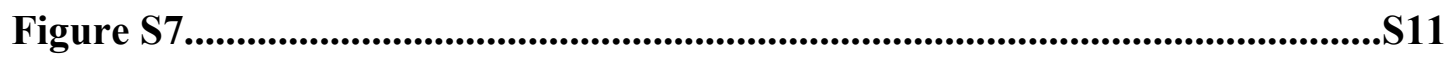

Supplementary Table

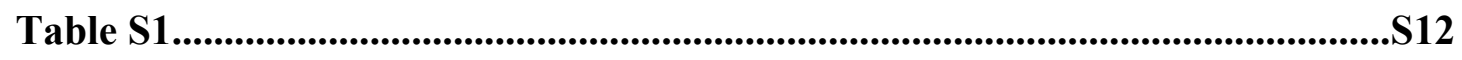

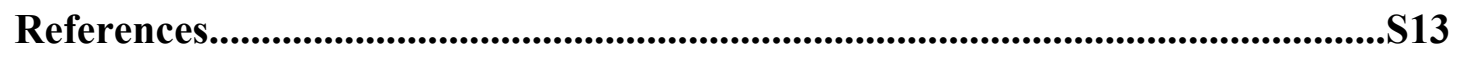




\section{Experimental Section}

\section{Chemicals and materials.}

Mouse anti-CD63 monoclonal antibody (Ab-CD63, >95\%), rabbit anti-EpCAM polyclonal antibody (Ab-EpCAM, >95\%), rabbit anti-CD9 polyclonal antibody (AbCD9, $>95 \%$ ) and rabbit anti-CD44 polyclonal antibody (Ab-CD44, >95\%), horseradish peroxidase (HRP)-conjugated goat anti-rabbit IgG were from Biosynthesis Biotechnology (Beijing, China). 3,3',5,5'-tetramethylbenzidine (TMB), trypsin-EDTA, and penicillin-streptomycin were from Beijing Solarbio Science \&Technology Co., Ltd. (Beijing, China). MCF-7 cell and MDA-MB231 cell were from Shanghai Institute of Biochemistry and Cell Biology. Dulbecco's modified eagle's medium (DMEM), fetal bovine serum (FBS) and phosphate buffered saline (PBS) were from Gibco. Antibody linker, $\mathrm{SH}\left(\mathrm{CH}_{2}\right)_{11}\left(\mathrm{OCH}_{2} \mathrm{CH}_{2}\right)_{6} \mathrm{OCH}_{2} \mathrm{COOH}$, was from Senso Path technologies (MT, USA). Mass tag, $\left[\mathrm{S}\left(\mathrm{CH}_{2}\right)_{11}\left(\mathrm{OCH}_{2} \mathrm{CH}_{2}\right)_{4} \mathrm{OH}\right]_{2}$, bovine serum albumin (BSA, $\geq 98 \%$ ), Nhydroxysuccinimide (NHS, 98\%), 1-ethyl-3 (3-dimethylaminopropyl) carbodiimidehydrochioride (EDC, $>98 \%$ ) and 2-(N-morpholino) ethanesulfonic acid (MES) were from Sigma-Aldrich. 6-Mercapto-1-hexanol (MCH, >98\%) was from TCI Co. Ltd. (Shanghai, China). $\mathrm{HAuCl}_{4} \cdot 4 \mathrm{H}_{2} \mathrm{O}, \mathrm{NaH}_{2} \mathrm{PO}_{4}$ and $\mathrm{Na}_{2} \mathrm{HPO}_{4}$ were from Sinopharm Chemical Reagent Co. Ltd. (Shanghai, China). Ultrapure water $(18.2 \mathrm{M} \Omega \cdot \mathrm{cm})$ used in all experiments was obtained from a Milli-Q system (Millipore, Bedford, MA). All solvents were used as received without further purification.

\section{Cell culture and exosome isolation.}

Breast cancer cells (MCF-7, MDA-MB231) were cultured in DMEM supplemented with $10 \%(\mathrm{v} / \mathrm{v})$ FBS and $1 \%(\mathrm{v} / \mathrm{v})$ penicillin-streptomycin at $37{ }^{\circ} \mathrm{C}$ in a humidified atmosphere with $5 \% \mathrm{CO}_{2}$. When $80 \%$ confluency was achieved, the cells were washed twice with PBS and then incubated in a serum-free medium for $24 \mathrm{~h}$.

Exosomes were isolated by ultracentrifugation method as reported. ${ }^{1}$ Briefly, the supernatant harvested from conditioned medium was centrifuged at $2000 \mathrm{~g}$ for $15 \mathrm{~min}$ to remove cell debris. To obtain the exosomes, the supernatant was filtered through a 
$0.22 \mu \mathrm{m}$ filter and then centrifuged at $120000 \mathrm{~g}$ for $3 \mathrm{~h}$. After washing with PBS, the exosomes were stored in PBS at $-80{ }^{\circ} \mathrm{C}$. All the centrifugal processes were kept at $4{ }^{\circ} \mathrm{C}$.

\section{Characterization of exosome.}

For TEM characterization, the isolated exosomes were dropped on $\mathrm{Cu}$ grids and dried at room temperature, then stained by $1 \%$ phosphotungstic acid. The TEM were performed on a JEOL JEM-2100 instrument (JEOL Ltd., Japan).

For Western blot analysis, exosomes were firstly lysed by radio-immunoprecipitation (RIPA) buffer containing protease inhibitors. The obtained exosomal proteins were quantified by bicinchoninic acid assay (BCA) and electrophoresed by SDS-PAGE. Then, the gels were transferred to nitrocellulose filter membranes and blocked with 5\% BSA. Furthermore, the membranes were incubated with Ab-CD63 overnight at $4{ }^{\circ} \mathrm{C}$, followed by washing, and incubated with HRP-conjugated secondary antibody for $1 \mathrm{~h}$. The bands were observed by a Gel imaging system (BioRad electrophoresis apparatus and imaged on a Typhoon 9410 variable mode imager (Amersham, USA).

\section{Screening exosome protein biomarkers by ELISA.}

The isolated exosomes (50 $\mu \mathrm{L} /$ well) were incubated in 96-well microtiter plates for 12 h at $4{ }^{\circ} \mathrm{C}$. After washing 3 times with PB, the plates were blocked with 1\% BSA (100 $\mu \mathrm{L} /$ well) in PB overnight at $4{ }^{\circ} \mathrm{C}$. Then, $2 \mu \mathrm{g} / \mathrm{mL}$ of primary antibodies against EpCAM, CD9 or CD44 (100 $\mu \mathrm{L} /$ well) were added and incubated for $1 \mathrm{~h}$ at $37^{\circ} \mathrm{C}$, followed by the addition of HRP-conjugated secondary antibody (1:40000, $100 \mu \mathrm{L} /$ well), and then incubated for $1 \mathrm{~h}$ at $37^{\circ} \mathrm{C}$. Subsequently, $100 \mu \mathrm{L} /$ well of TMB was added, and the reaction was stopped after $30 \mathrm{~min}$ by adding $2 \mathrm{M} \mathrm{H}_{2} \mathrm{SO}_{4}(50 \mu \mathrm{L} /$ well). The plates were measured at $450 \mathrm{~nm}$ using a microplate reader (Multiscan FC, Thermo Fisher Scientific Co., Ltd., USA). The 96-well microtiter plates were thoroughly washed between each step. 


\section{Supplementary Figure}
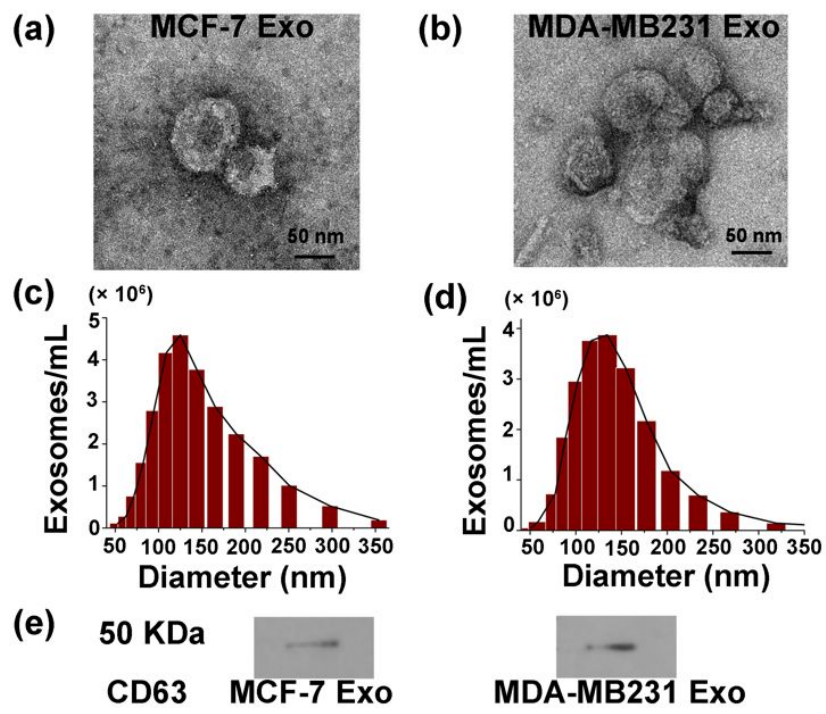

Figure S1. Characterization of the exosomes isolated from MCF-7 and MDA-MB231 cells by ultracentrifugation. TEM images of (a) MCF-7 Exo and (b) MDA-MB231 Exo. Size distribution of (c) MCF-7 Exo and (d) MDA-MB231 Exo measured by NTA. (e) Western blot images of the CD63 protein from MCF-7 Exo and MDA-MB231 Exo. 


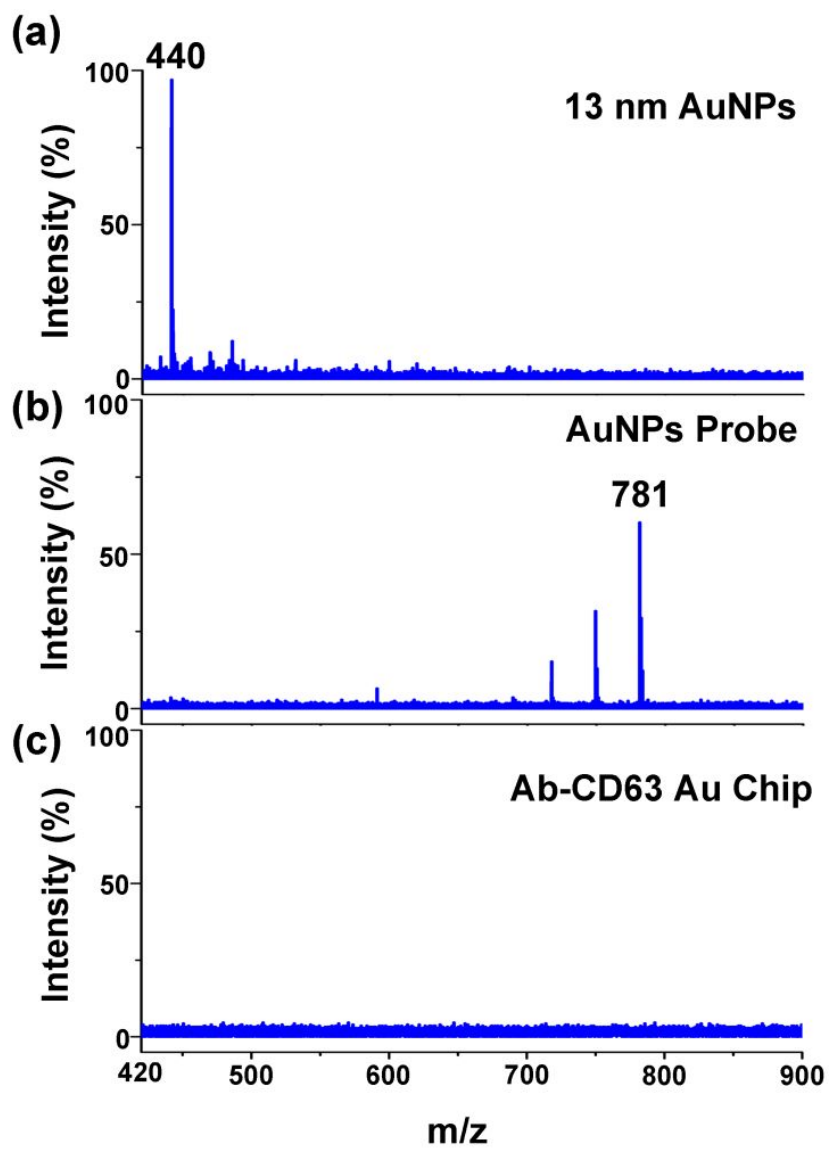

Figure S2. Mass spectra of AuNPs (a) before and (b) after modified with mass tags as well as (c) $\mathrm{Ab}-\mathrm{CD} 63 \mathrm{Au}$ chip. The peak at $\mathrm{m} / \mathrm{z} 440$ is assigned to $\left([\mathrm{Au}+\mathrm{Na}]_{2}\right)^{+}$. The peak at $\mathrm{m} / \mathrm{z} 781$ is assigned

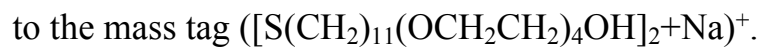




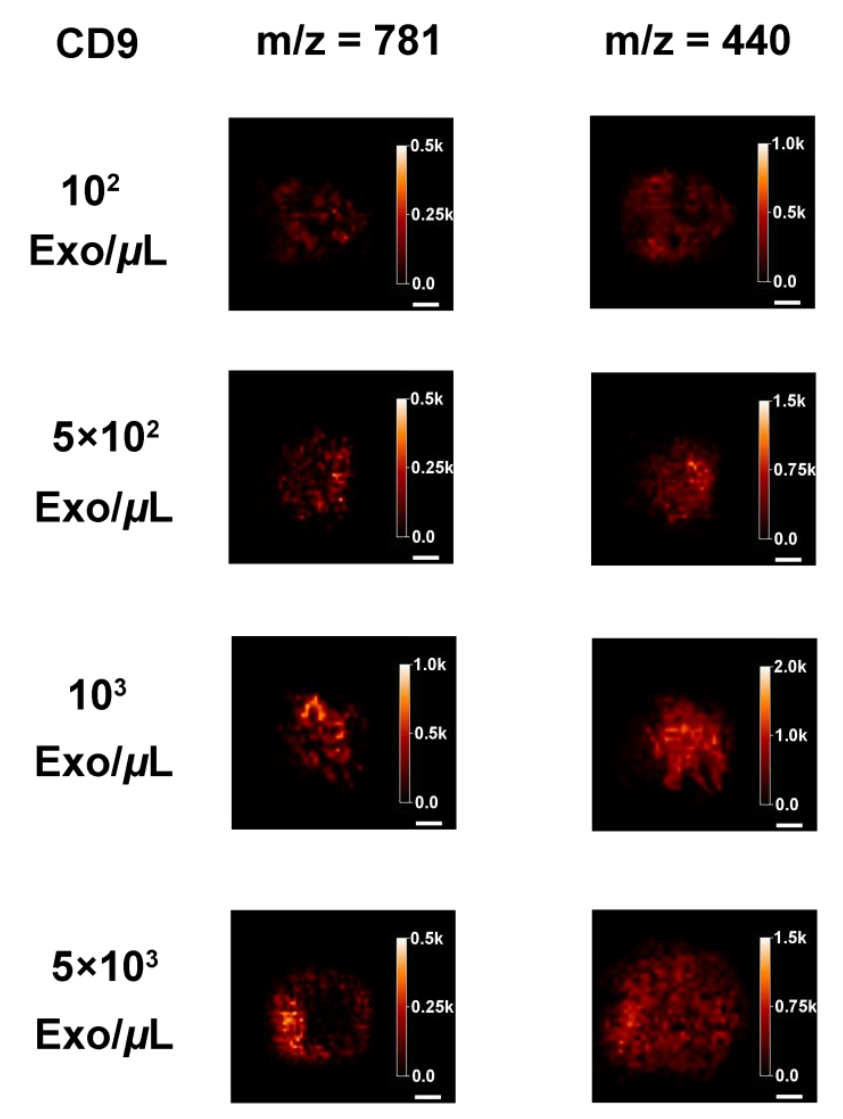

Figure S3. MSI mapping of CD9 on the surface of MCF-7 Exo with the concentration of exosome ranging from 100 to $5 \times 10^{3}$ particles $/ \mu \mathrm{L}$ ( $5 \mu \mathrm{L}$ sample loading amount). Images of the mass tag and IS distribution on the chips corresponding to the MS peaks at $\mathrm{m} / \mathrm{z} 781$ and 440 . The scale bar is 0.5 $\mathrm{mm}$. 


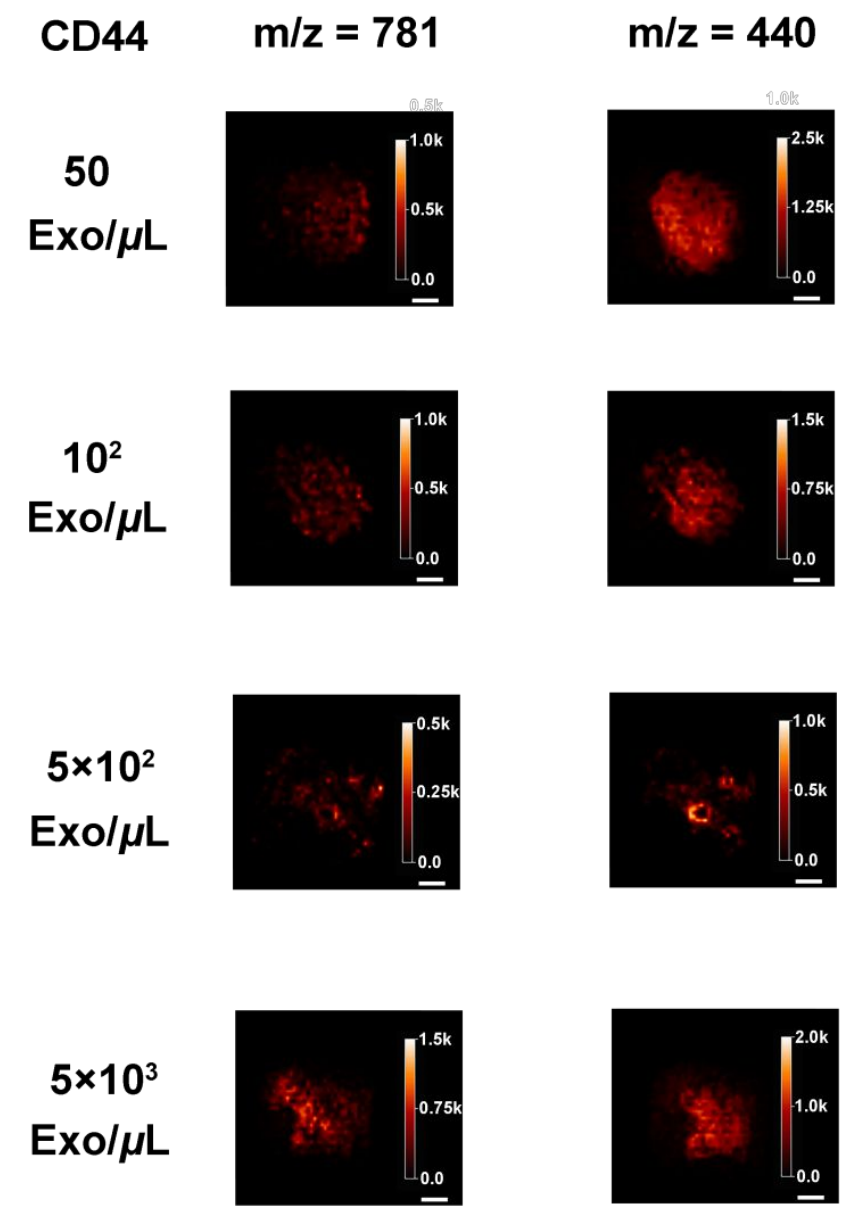

Figure S4. MSI mapping of CD44 on the surface of MCF-7 Exo with the concentration of exosome ranging from 50 to $5 \times 10^{3}$ particles $/ \mu \mathrm{L}$ ( $5 \mu \mathrm{L}$ sample loading amount). Images of the mass tag and IS distribution on the chips corresponding to the peaks at $\mathrm{m} / \mathrm{z} 781$ and 440 . The scale bar is $0.5 \mathrm{~mm}$. 


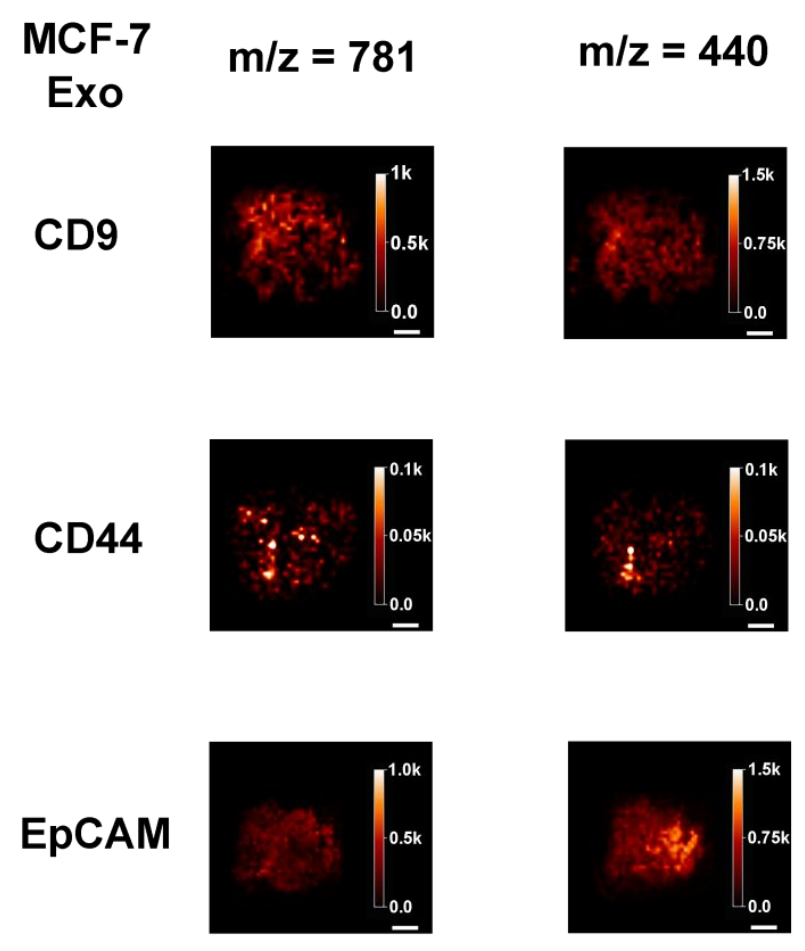

Figure S5. MSI signal amplification profiling of various protein biomarkers on the surface MCF-7 Exo with the concentration of $10^{4}$ particles $/ \mu \mathrm{L}$ ( $5 \mu \mathrm{L}$ sample loading). Images of the mass tag and IS distribution on the chips corresponding to the peaks at $\mathrm{m} / \mathrm{z} 781$ and 440 . The scale bar is $0.5 \mathrm{~mm}$. 


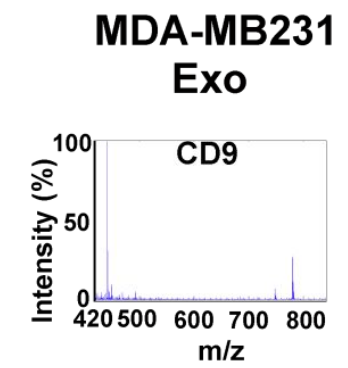

$$
\mathrm{m} / \mathrm{z}=\mathbf{7 8 1} \mathrm{m} / \mathrm{z}=\mathbf{4 4 0}
$$
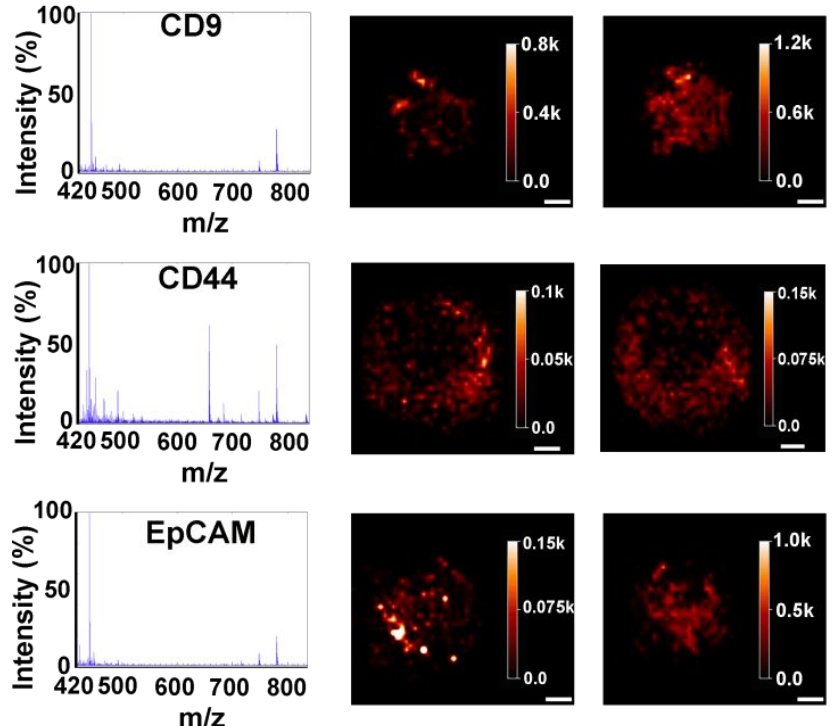

Figure S6. MSI signal amplification profiling of various protein biomarkers on the surface MDAMB231 Exo with the concentration of $10^{4}$ particles $/ \mu \mathrm{L}$ ( $5 \mu \mathrm{L}$ sample loading). (a) The integral average mass spectra and (b) Images of the mass tag and IS distribution on the chips corresponding to the peaks at $\mathrm{m} / \mathrm{z} 781$ and 440 . The scale bar is $0.5 \mathrm{~mm}$. 


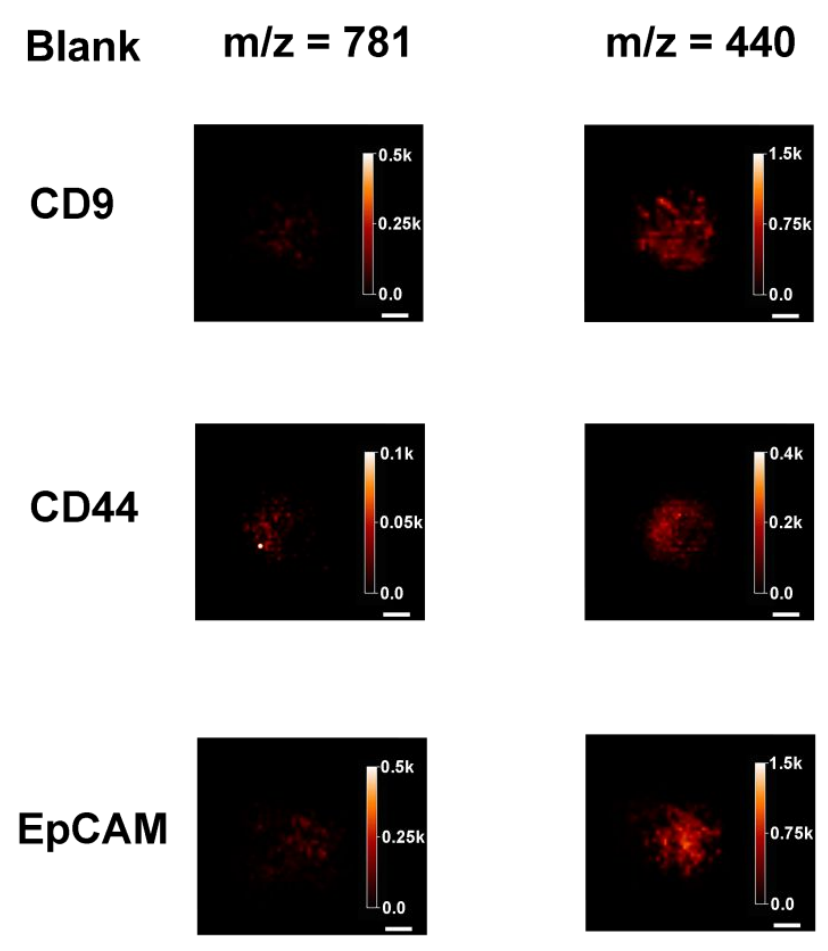

Figure S7. MSI signal amplification profiling of various protein biomarkers from a blank control sample. Images of the mass tag and IS distribution on the chips corresponding to the peaks at $\mathrm{m} / \mathrm{z}$ 781 and 440. The scale bar is $0.5 \mathrm{~mm}$. 


\section{Supplementary Table}

Table S1. Comparison of different methods for exosomal proteins detection

\begin{tabular}{|c|c|c|c|}
\hline Platform & Detection Method & $\begin{array}{l}\text { LOD } \\
\text { (Exosome } \\
\text { particles) }\end{array}$ & References \\
\hline $\begin{array}{l}\text { Quantitative Localized Analysis } \\
\text { (QLA) }\end{array}$ & Electrochemistry & $\sim 10^{4}$ & 2 \\
\hline $\begin{array}{l}\text { Integrated magnetic- } \\
\text { electrochemical exosome (iMEX) }\end{array}$ & Electrochemistry & $\sim 10^{4}$ & 3 \\
\hline $\begin{array}{l}\text { Exosome-oriented, aptamer } \\
\text { nanoprobe (ExoAPP) }\end{array}$ & Fluorescence & $\sim 10^{3}$ & 4 \\
\hline Thermophoretic aptasensor (TAS) & Fluorescence & $\sim 10^{3}$ & 5 \\
\hline nano-Plasmonic Exosome (nPLEX) & $\begin{array}{l}\text { Surface Plasmon } \\
\text { Resonance }\end{array}$ & $\sim 10^{3}$ & 6 \\
\hline EXBioMChip & Mass Spectrometry & $\sim 50$ & This work \\
\hline
\end{tabular}




\section{References}

(1) Li, T.-D.; Zhang, R.; Chen, H.; Huang, Z.-P.; Ye, X.; Wang, H.; Deng, A.-M.; Kong, J.-L. An Ultrasensitive Polydopamine Bi-Functionalized SERS Immunoassay for Exosome-Based Diagnosis and Classification of Pancreatic Cancer. Chem. Sci. 2018, 9, 5372-5382.

(2) Guo, Y.; Tao, J.; Li, Y.; Feng, Y.; Ju, H.; Wang, Z.; Ding, L. Quantitative Localized Analysis Reveals Distinct Exosomal Protein-Specific Glycosignatures: Implications in Cancer Cell Subtyping, Exosome Biogenesis, and Function. J. Am. Chem. Soc. 2020, 142, 7404-7412.

(3) Jeong, S.; Park, J.; Pathania, D.; Castro, C. M.; Weissleder, R.; Lee, H. Integrated MagnetoElectrochemical Sensor for Exosome Analysis. ACS Nano 2016, 10, 1802-1809.

(4) Liu, C.; Zhao, J.; Tian, F.; Cai, L.; Zhang, W.; Feng, Q.; Chang, J.; Wan, F.; Yang, Y.; Dai, B.; Cong, Y.; Ding, B.; Sun, J.; Tan, W. Low-Cost Thermophoretic Profiling of Extracellularvesicle Surface Proteins for the Early Detection and Classification of Cancers. Nat. Biomed. Eng. 2019, 3, 183-193.

(5) Jin, D.; Yang, F.; Zhang, Y.; Liu, L.; Zhou, Y.; Wang, F.; Zhang, G.-J. ExoAPP: ExosomeOriented, Aptamer Nanoprobe-Enabled Surface Proteins Profiling and Detection. Anal. Chem. 2018, 90, 14402-14411.

(6) Im, H.; Shao, H.; Park, Y. I.; Peterson, V. M.; Castro, C. M.; Weissleder, R.; Lee, H. LabelFree Detection and Molecular Profiling of Exosomes with a Nano-Plasmonic Sensor. Nat. Biotechnol. 2014, 32, 490-495. 